\title{
RNA-Seq analysis reveals that multiple phytohormone biosynthesis and signal transduction pathways are reprogrammed in curled-cotyledons mutant of soybean [Glycine max (L.) Merr.]
}

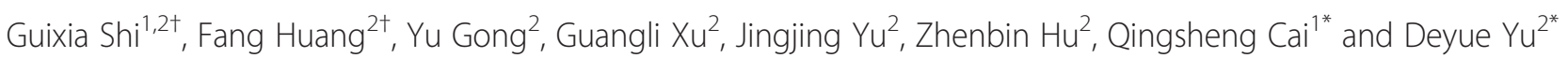

\begin{abstract}
Background: Soybean is one of the most economically important crops in the world. The cotyledon is the nutrient storage area in seeds, and it is critical for seed quality and yield. Cotyledon mutants are important for the genetic dissection of embryo patterning and seed development. However, the molecular mechanisms underlying soybean cotyledon development are largely unexplored.
\end{abstract}

Results: In this study, we characterised a soybean curled-cotyledon (cco) mutant. Compared with wild-type (WT), anatomical analysis revealed that the cco cotyledons at the torpedo stage became more slender and grew outward. The entire embryos of cco mutant resembled the "tail of swallow". In addition, cco seeds displayed reduced germination rate and gibberellic acid $\left(G_{3}\right)$ level, whereas the abscisic acid (ABA) and auxin (IAA) levels were increased. RNA-seq identified 1,093 differentially expressed genes (DEGs) between WT and the cco mutant. The KEGG pathway analysis showed many DEGs were mapped to the hormone biosynthesis and signal transduction pathways. Consistent with assays of hormones in seeds, the results of RNA-seq indicated auxin and $A B A$ biosynthesis and signal transduction in cco were more active than in WT, while an early step in GA biosynthesis was blocked, as well as conversion rate of inactive GAs to bioactive GAs in GA signaling. Furthermore, genes participated in other hormone biosynthesis and signalling pathways such as cytokinin $(\mathrm{CK})$, ethylene (ET), brassinosteroid (BR), and jasmonate acid (JA) were also affected in the cco mutant.

Conclusions: Our data suggest that multiple phytohormone biosynthesis and signal transduction pathways are reprogrammed in $\mathrm{cco}$, and changes in these pathways may partially contribute to the cco mutant phenotype, suggesting the involvement of multiple hormones in the coordination of soybean cotyledon development.

Keywords: Soybean, RNA-seq, Plant hormone, Curled-cotyledon, Mutant

\footnotetext{
*Correspondence: qscai@njau.edu.cn; dyyu@njau.edu.cn

${ }^{\dagger}$ Equal contributors

${ }^{1}$ College of Life Sciences, Nanjing Agricultural University, Nanjing, China

${ }^{2}$ National Center for Soybean Improvement, National Key Laboratory of Crop

Genetics and Germplasm Enhancement, Nanjing Agricultural University,

Nanjing, 210095, China
}

\section{Biomed Central}

(c) 2014 Shi et al.; licensee BioMed Central Ltd. This is an Open Access article distributed under the terms of the Creative Commons Attribution License (http://creativecommons.org/licenses/by/2.0), which permits unrestricted use, distribution, and reproduction in any medium, provided the original work is properly credited. The Creative Commons Public Domain Dedication waiver (http://creativecommons.org/publicdomain/zero/1.0/) applies to the data made available in this article, unless otherwise stated. 


\section{Background}

Cotyledon is the main nutrient storage area in soybean seed, which contains approximately $40 \%$ protein and $20 \%$ oil at maturity [1]. Flowering plants are divided into monocots and dicots based on the number of cotyledons. In Arabidopsis, as a paradigm for dicot embryonic development, cotyledons are specified from two lateral domains at the apical end of the embryo proper at the heart stage, showing bilateral symmetry [2]. Several studies have reported cotyledon mutants in Arabidopsis [3-7], Antirrhinum [8], tomato [9-11], and other plants [12]. These mutants have been used to identify a number of orthologous gene hierarchies that are involved in cotyledon development [13]. The Arabidopsis cup-shaped cotyledon (CUC) genes (CUC1, CUC2, and CUC3), belonging to the plant-specific NAC transcription factor, Picea glauca NAC01, and SHOOT MERISTEMLESS (STM) are required for shoot apical meristem (SAM) formation and cotyledon separation [4,14-16]. STM induces the expression of $C U C$ genes, and the induction is specific and independent of other meristem regulators [17]. STM negatively regulates ASYMMETRIC LEAVES1 (AS1), and the mutation of AS1 in Arabidopsis disrupts cotyledon development [18]. KNOTTED-like (KNAT6) is expressed at the boundary between the SAM and cotyledon later than STM and CUC, suggesting that KNAT6 plays a crucial role in SAM maintenance and boundary establishment in embryos via the STM/CUC pathway [19].

Phytohormones, primarily auxin, CK, BR, GA, ABA, and JA, orchestrate intrinsic developmental programs. Previous studies have greatly advanced the functions of individual hormone. During last two decades, extensive lists of genes involved in hormone synthesis, catabolism and signal transduction have been identified via the analysis of mutants [20-23]. Auxin plays a key regulatory role in the initiation of cotyledon in the apical margin of the globular embryo [24]. In many plant species, including Brassica napus [25], Arabidopsis [26], and Norway spruce [27], studies have shown that cotyledon and SAM development could be inhibited by various polar auxin inhibitors in embryogenesis. PIN auxin efflux regulators are the best characterised components of auxin transport. The polar location of PIN1 is associated with the position of auxin accumulation in incipient cotyledons, and auxin flows into the primordium interior during cotyledon outgrowth [28]. The pin1 pid double mutant completely lacks cotyledons and bilateral symmetry and exhibits increased CUC1, CUC2 and STM expression, indicating that directional auxin transport is important for the establishment of bilateral symmetry and the promotion of cotyledon outgrowth [26]. Similarly, the pid enp double mutant, which is similar to "laterne", also abolishes cotyledon development, reflecting failed auxin accumulation in the apex, and ENP is necessary for cotyledon development via the control of PIN1 polarity in the context of PID [29]. In addition to auxin, some studies have shown that CK affects cotyledon development. Exogenous application of benzyladenine (the synthetic cytokinin) onto hybrid larch somatic embryos reduced the cotyledon number [30]. The amp-arabidopsis mutant demonstrated polycotyly, indicating an elevated cytokinin level [31]. Arabidopsis ESR1 and ESR2, whose expression levels are induced by cytokinin, not only control shoot regeneration but also play a role in cotyledon development $[32,33]$.

In this study, we characterised a soybean mutant named $c c o$, which displayed curled cotyledons. The $c c o$ embryos at the torpedo stage resembled the "tail of swallow". High performance liquid chromatography (HPLC) analysis showed that $c c o$ seeds contained higher IAA and ABA levels, but lower $\mathrm{GA}_{3}$ level. Transcriptome analysis revealed that multiple phytohormone biosynthesis and signal transduction pathways were reprogrammed in $c c o$.

\section{Results and discussion}

\section{Embryogenesis in the cco mutant}

We obtained the cco mutant following sodium azide $\left(\mathrm{NaN}_{3}\right)$ and ${ }^{60} \mathrm{Coy}$ ray seed mutagenesis of soybean [34], which was initially characterised by outward folding cotyledons (Figure 1A-C, $1 \mathrm{M}$ and $1 \mathrm{~N}$ ). Compared with WT, developing cotyledons of $c c o$ mutant had larger vacuoles and more membranous multilamellar structures, as well as higher methionine and cysteine content [34]. In addition, the germination rates of seeds were compared between the cco mutant and WT. In general, seeds from WT germinated on the second day, and the germination rate reached $\sim 72 \%$ on the fifth day. cco seeds were dormant until the third day, and the germination rate was only $\sim 6.7 \%$ on fifth day, suggesting that the germination rate of $c c o$ seeds was greatly reduced (Figure 1O).

To determine the embryogenesis defects in $c c o$ mutant, we traced its phenotype back to the globular stage of embryogenesis. In higher plants, embryogenesis can be conceptually divided into three overlapping phases: morphogenesis, maturation and desiccation [35]. In general, for the wild type at the globular stage, an embryo comprises a spherical embryo proper and a suspensor (Figure 1D). The embryo proper is spherical during the pro-embryo and globular stage and eventually forms cotyledons, shoot meristem and hypocotyl region around the apical-basal axis, and this structure is connected to the embryo sac via the suspensor. During the globular-heart transition phase, a dramatic change occurs: two of the cotyledon primordia are symmetrically initiated from the lateral domains at the apical end of the heart-shaped embryo proper (Figure 1E). Subsequently, the cotyledons and hypocotyl elongate, and the embryo enters the torpedo stage in which the cotyledons continue to rotate. The morphogenesis phase of embryogenesis ends at the cotyledon stage (Figure $1 \mathrm{~F}$ and $1 \mathrm{G}$ ). 


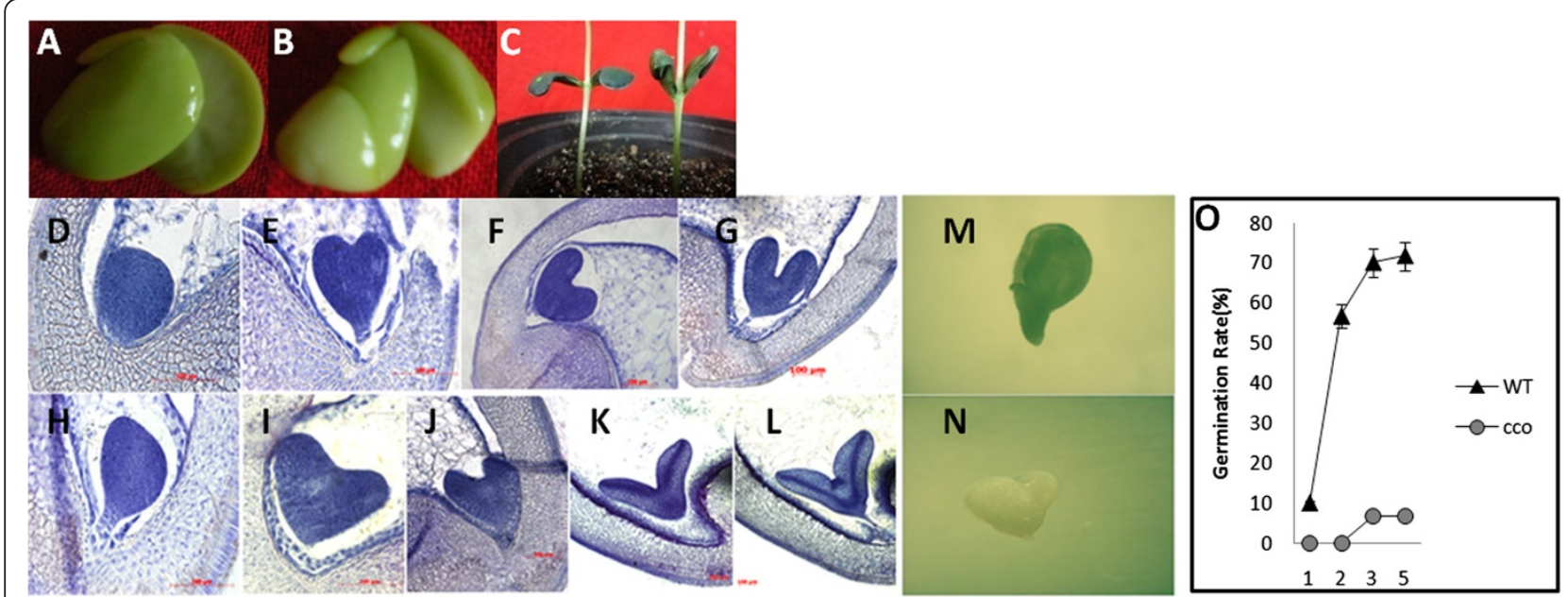

Figure 1 Embryogenesis of the cco mutant. (A) Cotyledons of WT. (B) Cotyledons of the cco mutant. (C) Cotyledons after germination for 5 days. WT (left) and the cco mutant (right). (D) WT embryo at the globular stage approximately 9 days after fertilisation. (E) WT embryo at the heart stage approximately 11 days after fertilisation. (F) and (G) WT embryo at the torpedo stage approximately 13 days after fertilisation. (H) cco embryo at the globular stage approximately 11 days after fertilisation. (I) cco embryo at the heart stage approximately 13 days after fertilisation. (J) to (L) cco embryos at the different torpedo stages approximately 15 days after fertilisation. Bar $=100 \mu \mathrm{m}$ for (D) to (L). (M) WT embryo at the cotyledon stage. (N) cco embryo at the cotyledon stage. (0) Germination rates of WT and cco. WT, triangles; cco, circles. ${ }^{* *} P<0.01$.

However, the development of embryos in cco was slower than that in WT (Figure 1). When WT embryos were at the heart stage, cco embryos remained morphologically at the globular stage (Figure $1 \mathrm{E}$ and $1 \mathrm{H}$ ). The embryo proper in cco appeared smaller than that in WT (Figure $1 \mathrm{D}$ and $1 \mathrm{H}$ ). At the heart stage, cotyledons were initiated from the cotyledon primordia. Figure $1 \mathrm{E}$ and $1 \mathrm{I}$ showed that the cotyledon initiation positions were identical in both the WT and cco. However, the WT cotyledons grew upwards, while that of the cco mutant demonstrated outward growth. Subsequently, the bifurcation of the cotyledons at the torpedo stage in the cco mutant was larger, and the tips of the cotyledons were sharper (Figure $1 \mathrm{~F}$ to $1 G$ and $1 \mathrm{~J}$ to $1 \mathrm{~L})$. At the torpedo stage, cco mutant embryos resembled "tail of swallow", while the radicle and shoot apical meristem were also present and appeared normal. All in all, cco showed abnormal embryogenesis, especially at the torpedo stage, when the cco cotyledons became more slender and grew outward. However, SAM and the polar axis were normal. Cotyledons and leaves share homology in plants [13]. Cotyledon initiation and development in cco were defective, while leaf development was correct, suggesting partial homology between leaf and cotyledon.

Increased free IAA and ABA levels, while decreased $\mathrm{GA}_{3}$ level in cco

The hormone auxin governs a variety of developmental processes [36], including embryogenesis [37]. In embryogenesis, auxin controls cell division and specification, which are critical for establishing the embryo pattern.
Most of the pattern formation events in Arabidopsis embryogenesis, including cotyledon development, depend on auxin biosynthesis, transport, and response [37]. IAA is the most important natural auxin. Therefore, we examined the endogenous free IAA level in WT and the cco mutant seeds at 7 DAF (days after fertilisation) by HPLC analysis (Figure 2). Compared with WT, the cco mutant had a higher free IAA level in seeds at 7 DAF.

In addition, the endogenous GA3 and ABA content in WT and cco seeds at 7 DAF were quantified. The measurements demonstrated that the ABA content was significantly increased, while the GA3 content was decreased in $c c o$ (Figure 2). The increased ABA/GA ratio may lead to a low germination rate for the $c c o$ mutant.

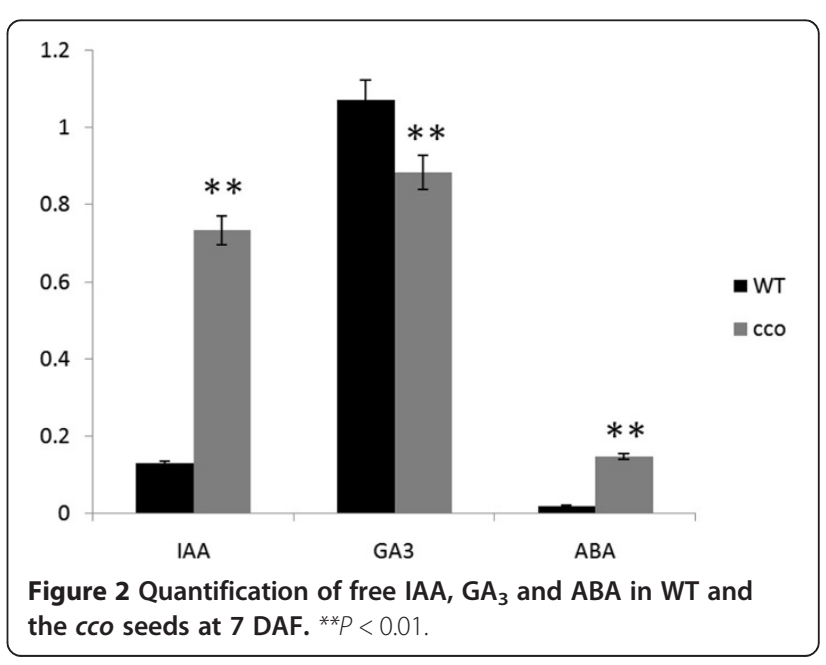




\section{Auxin sensitivity was depressed in cco}

Twelve auxin responsive genes, including two AUX/IAA genes (Glyma08g22190, Glyma10g32340), five GH3 genes (Glyma02g13910, Glyma02g17360, Glyma11g05510, Glyma12g11890, Glyma12g11200) and five SAUR genes (Glyma10g06360, Glyma10g06370, Glyma05g36360, Glyma12g03780, Glyma09g35460), were selected to investigate the transcription of early auxin-regulated genes in response to auxin treatment by quantitative RT-PCR (qRT-PCR). As shown in Figure 3, all of these genes were up-regulated in WT at $30 \mathrm{~min}$ after treatment with $0.05 \mu \mathrm{M}$ 2,4-dichlorophenoxyacetic acid (2,4-D). However, in the cco mutant, only three genes (Glyma08g22190, Glyma12g11200, and Glyma09g35460) were weakly up-regulated, and the other genes had weak variation or even unexpected down-regulation (Figure 3A). When hypocotyls were treated with $0.2 \mu \mathrm{M} 2,4-\mathrm{D}$, the twelve genes tested showed higher expression levels in WT, and Glyma08g22190 and Glyma11g05510 showed greater than five-fold up-regulation (Figure 3B). With the exception of Glyma12g11890 and Glyma05g36360, the expression trends of the genes in $c c 0$ were similar to that observed after treatment with $0.05 \mu \mathrm{M}$ 2,4-D (Figure 3). Thus, auxin sensitivity were repressed in the $c c o$ mutant.

\section{RNA-Seq of WT and the cco mutant}

The positions of the cotyledon primordia in $c c o$ were generally normal, but the abaxial/adaxial patterning of cotyledons was flawed, which was likely to exist before cotyledon initiation. Thus, we focused on the globular stage, and the pods at 7 DAF were collected for RNA-Seq using the Illumina HiSeq2000 system. A total of 52,870,578 and 50,494,652 successful reads (average length: $~ 180 \mathrm{bp}$, approximately $4 \times$ soybean genome) were produced for WT and the $c c o$ mutant, respectively (Table 1). Notably, more than $85 \%$ of the reads mapped back to the soybean reference genome in Phytozome database [38]. In WT, 80.74\% of these genes uniquely mapped to a single location, and $80.27 \%$ uniquely mapped to a single location for cco (Table 1). We identified 39,499 highly confident genes in WT and 39,527 genes in the cco mutant. With a False Discovery Rate $(\mathrm{FDR})<0.001$ and $\mid \log _{2}$ Ratio $\mid \geq 1$, a total of 1,093 genes were differentially expressed; 256 DEGs had lower expression levels in the cco mutant, while 837 DEGs were activated (Figure 4 and Additional file 1). In this study, we primarily focused on the differentially expressed genes. The expression levels of the majority of these genes were unaffected in the cco mutant, suggesting that $c c o$ targeted a limited number of genes.

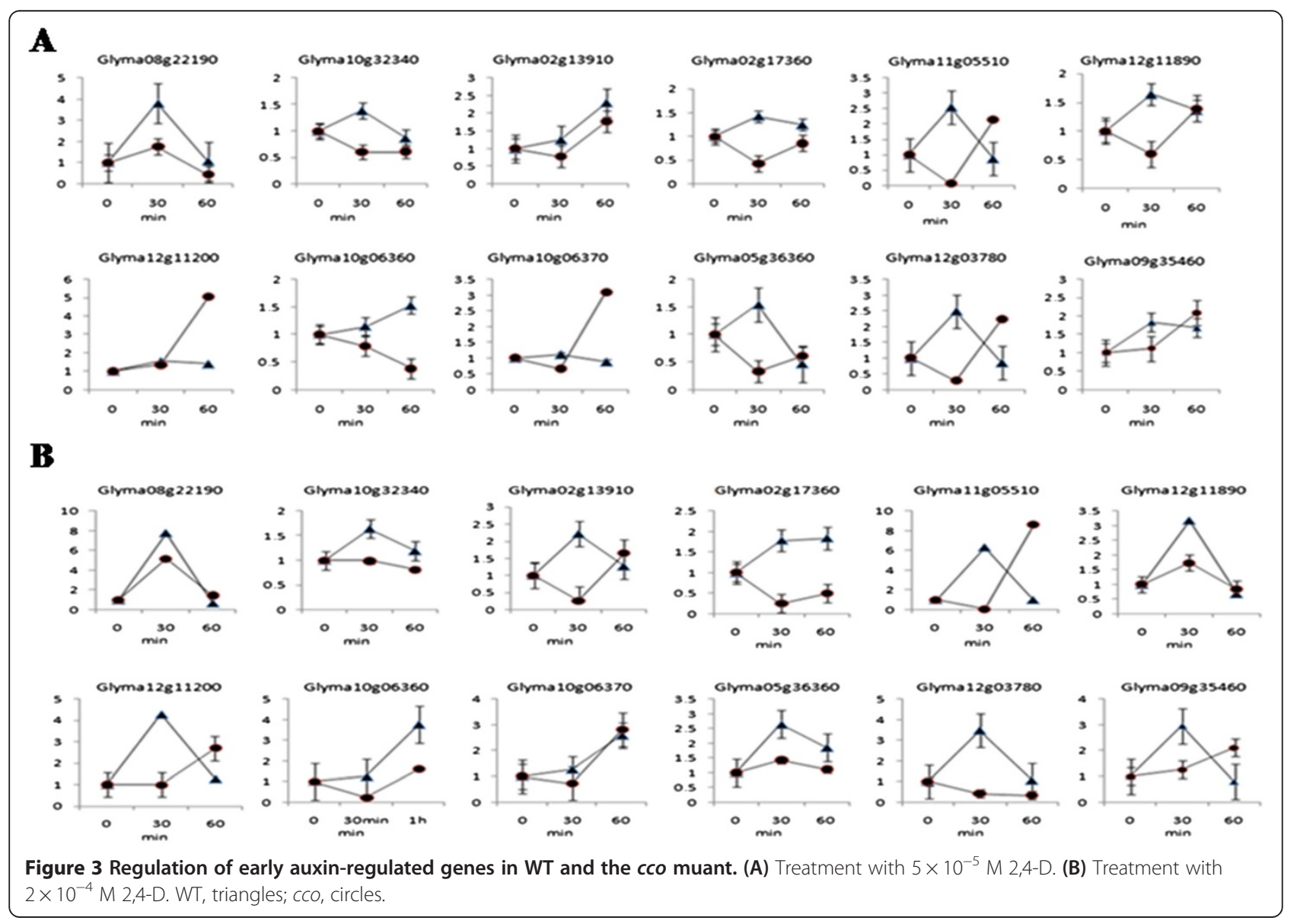


Table 1 Summary of RNA-seq data from WT and cco pods at 7 DAF

\begin{tabular}{lllllll}
\hline & Total reads & Total base pairs & Total mapped reads & Percent mapped & Unique match & Percent unique match \\
\hline WT & $52,870,578$ & $4,758,352,020$ & $45,064,067$ & $85.23 \%$ & 42687787 & $80.74 \%$ \\
cCO & $50,494,652$ & $4,544,518,680$ & $42,914,706$ & $84.99 \%$ & 40531216 & $80.27 \%$ \\
\hline
\end{tabular}

To validate the reliability of the expression profiles obtained using RNA-Seq, we selected fifteen genes with higher or lower expression levels for semi-quantitative reverse transcription PCR (RT-PCR) analysis (Table 2 and Additional file 2: Figure S1A and $\mathrm{S} 1 \mathrm{~B}$ ), and three (Glyma03g02620, Glyma14g39300, Glyma11g11580) of the fifteen genes for further qRT-PCR analysis in 7DAF pods from WT and the cco mutant (Figure 5A). For all of the genes, the results obtained from the semiquantitative RT-PCR/qRT-PCR analysis were consistent with data from RNA-Seq.

In addition, we used semi-quantitative RT-PCR to analyse the expression of four selected genes (Glyma03g02620, Glyma14g39300, Glyma11g11580, Glyma17g14710) in WT and $c c o$ seeds at 7 and 15 DAF (Additional file 2: Figure S1C). Using qRT-PCR, we examined the expression of three genes (Glyma03g02620, Glyma14g39300, Glyma11g11580) in the roots, stems, leaves, flowers and seeds at the various developmental stages of WT and $c c 0$, and the results showed these genes were differentially expressed in these tissues (Figure 5B), indicating some of the DEGs that we obtained were differentially expressed in other tissues.

Functional classification of differentially expressed genes To evaluate the potential functions of the DEGs between WT and the cco mutant, Gene Ontology (GO) categories were used to identify key processes for $c c o$ mutant. Detailed GO term annotations of the DEGs were categorised according to biological processes, molecular functions and cellular components. The GO categories for the set of DEGs (Figure 6) revealed that most of the encoded

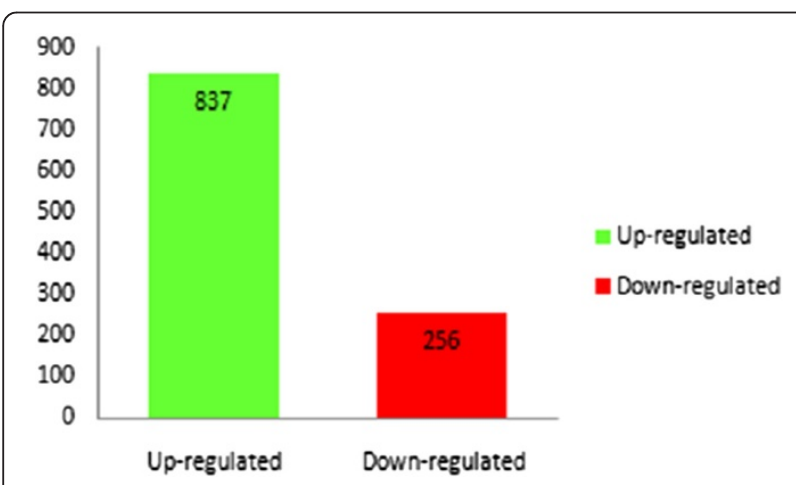

Figure 4 DEGs between WT and the cco mutant: the up- and down-regulated represent the genes that had an increased and decreased expression levels in cco. products were associated with "intracellular organelle", "catalytic activity", "response to stimulus" and "cellular metabolic process".

Biological interpretation of the 650 DEGs was further completed using KEGG pathway analysis. Many of the DEGs were mapped to pathways essential for plant growth and development. Overall, 98 pathways were affected in the cco mutant, and some of these pathways were consistent with biological processes previously revealed in the GO analysis. The most frequently represented pathways primarily involved in secondary metabolism, including plant hormone biosynthesis and signal transduction (20.46\%), such as auxin, CK, GA, ABA, ET, BR and JA (Additional file 3). Asakura et al. found that genes expressed in the early pod stage of soybean were the most numerous, compared with 2 and $5 \mathrm{~mm}$ seeds, and the number of expressed genes gradually decreased with seed development [39]. Commonly expressed genes in these stages accounted for $70-95 \%$ of the expressed genes in each stage, and only a small number of genes were differentially expressed, which mainly included $\beta$-conglycinin, lipid synthesis, lipoxygenase and seed-maturation proteins [39]. Therefore, most DEGs obtained in this study, including genes that were related to hormone biosynthesis and signalling, could still be identified in the early seed stage. However, some DEGs may have been missed because of tissue-dependent gene expression.

The results of the transcriptome data showed that many genes encoding proteins involved in auxin metabolism and signal transduction pathways were differentially expressed between $c c o$ and WT. L-tryptophan, as a primary precursor, is used to generate many indole-containing substances in plants, including IAA. $\alpha$-subunit anthranilate synthase (ASA) catalyses the conversion of chorismate to anthranilate as one of the first steps in the Trp biosynthesis pathway. In Arabidopsis, up-regulation of WEAK ETHYLENE INSENSITIVE2/ANTHRANILATE SYNTHASE $\alpha 1$ (WEI2/ $A S A 1)$ gene through ET results in auxin accumulation in the tips of primary roots [40]. Transgenic rice lines expressing $\alpha$-SUBUNIT OF ANTHRANILATE SYNTHASE (OASA1D) gene show increased production of tryptophan and free IAA [41]. Tryptophan synthase beta subunit (TSB) catalyses the last step in tryptophan biosynthesis. In IAA biosynthesis, indole-3-acetaldoximem (IAOx)-dependent IAA biosynthesis is a specific pathway in plants. In this process, two homologous cytochrome $\mathrm{P} 450$ enzymes, CYP79B2 and CYP79B3, mediate the synthesis of IAOx from tryptophan [42], and the double mutation of these two genes results in the downregulation of IAA synthesis. 
Table $\mathbf{2}$ Fold changes of $\mathbf{1 5}$ genes selected from RNA-seq data

\begin{tabular}{|c|c|c|c|c|}
\hline Gene ID & Gene description & Fold change & Regulation & P-value \\
\hline Glyma02g42290 & Transmembrane amino acid transporter protein & 2.02 & Up & 0.000139 \\
\hline Glyma03g09140 & Transmembrane amino acid transporter protein & 2.69 & Up & $2.58 \mathrm{E}-07$ \\
\hline Glyma05g23180 & Membrane transport protein & 5.08 & Up & $3.71 \mathrm{E}-05$ \\
\hline Glyma08g01430 & WRKY transcription factor & 12.62 & Up & $3.12 \mathrm{E}-06$ \\
\hline Glyma09g37370 & HLH transcription factor & 2.35 & Down & 2.84E-10 \\
\hline Glyma16g13570 & bZIP transcription factor & 2.03 & Up & 0.000139 \\
\hline Glyma18g05720 & NPH3 family & 2.34 & Down & $1.26 \mathrm{E}-16$ \\
\hline Glyma19g43761 & DUF640 & 4.56 & Up & 0.000186 \\
\hline Glyma17g14710 & YABBY protein & 3.05 & Up & $6.86 \mathrm{E}-24$ \\
\hline Glyma11g00580 & tryptophan synthase $\beta$-chain & 8.01 & Up & 1.85E-57 \\
\hline Glyma14g39300 & ubiquitin-protein ligase activity & 371.70 & Up & $3.14 \mathrm{E}-08$ \\
\hline Glyma03g02620 & LBD transcription factor & 441.39 & Down & $8.88 \mathrm{E}-05$ \\
\hline Glyma06g02970 & DBB transcription factor & 2.91 & Up & $9.21 \mathrm{E}-06$ \\
\hline Glyma06g17420 & HLH transcription factor & 2.06 & Up & $1.74 \mathrm{E}-68$ \\
\hline Glyma16g28310 & Fructose-1-6-bisphosphatase & 2.16 & Down & $2.39 \mathrm{E}-43$ \\
\hline
\end{tabular}

In the transcriptome data obtained in this study, one $A S A$ gene, one TBS gene and three CYP79 genes were upregulated, suggesting that auxin synthesis was enhanced in the cco mutant (Additional file 4). Mounting evidence suggests that polar auxin transport (PAT) controls important growth and developmental processes in higher plants. In Arabidopsis, studies have shown that the AUXIN1/LIKEAUX1 (AUX/LAX) family of auxin transporters are major influx carriers, whereas the PIN-FORMED (PIN) family of auxin proteins are major efflux carriers. The AUX/LAX

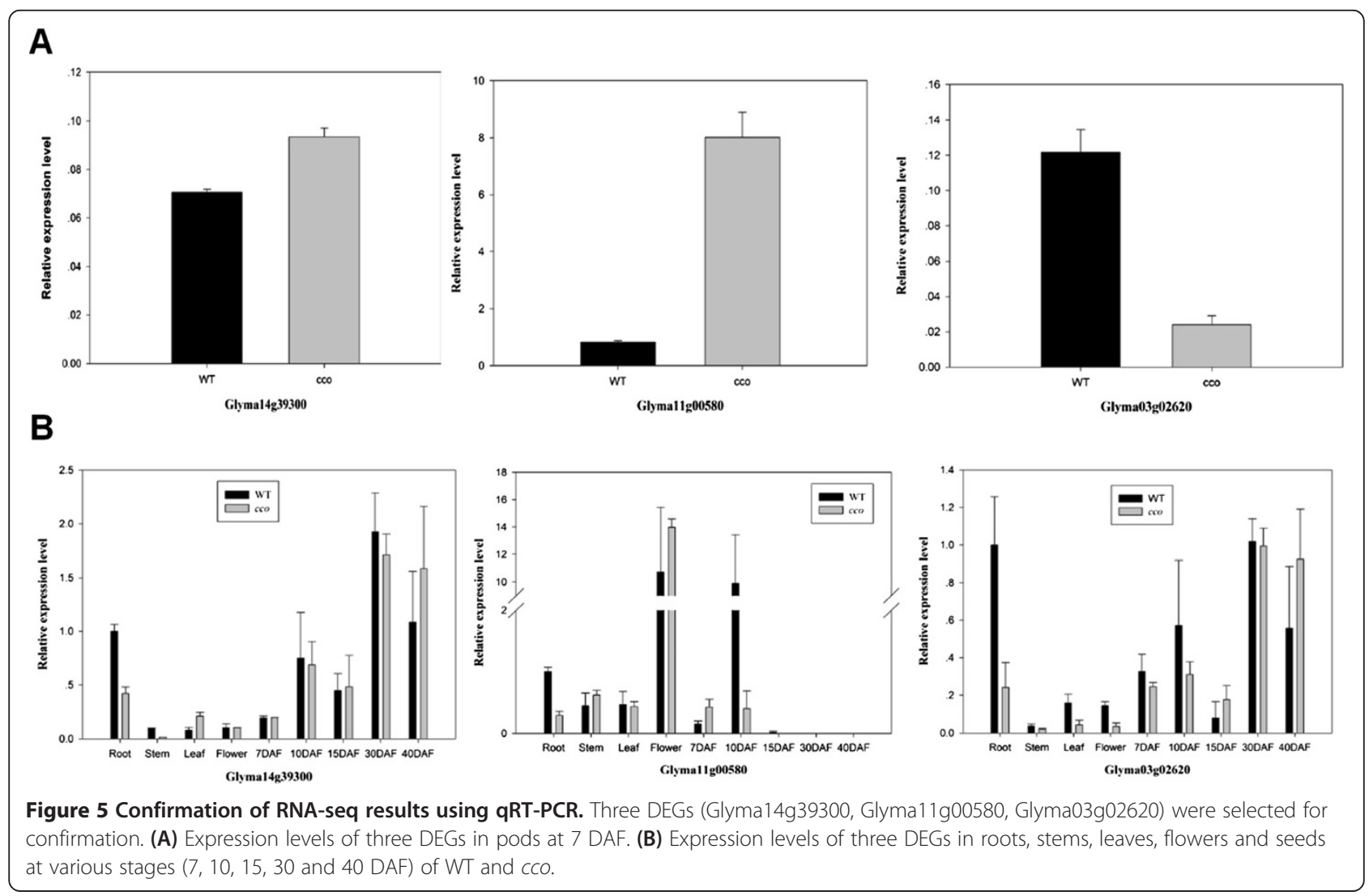




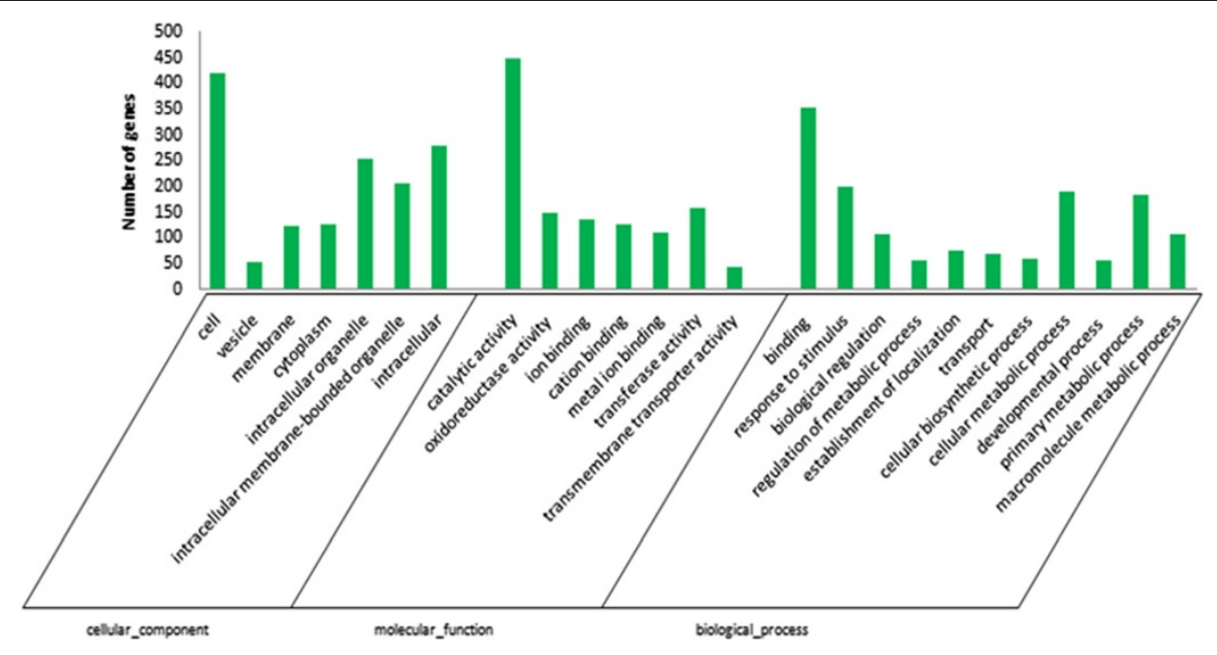

Figure 6 Gene Ontology classification of the differentially expressed genes between WT and cco. Differentially expressed genes are classified into three GO categories: cellular component, molecular function and biological process.

family is represented by four highly conserved genes, namely AUX1, LAX1,LAX2, and LAX3. LAX2 regulates vascular patterning in cotyledons. Transcriptome data showed that three LAX2 genes and one PIN gene were up-regulated in the $c c o$ mutant, indicating that auxin polar transport was also influenced. Auxin-responsive genes include GH3 genes, small auxin up RNA (SAUR) genes and $A U X / I A A$ genes [43]. In our transcriptome data, one $A U X / I A A$ and five SAUR genes were down-regulated, and four SAUR genes were up-regulated in $c c o$ (Figure 7). All of the GH3 genes were up-regulated, which were likely due to the down-regulation of the $A U X / I A A$ genes as Aux/lAA proteins repress the expression of $G H 3$ genes in soybean [44]. Taken together, these data indicated that auxin biosynthesis and signal transduction in $c c o$ were more active than in WT.

The role of GA in embryo morphogenesis has not yet been determined. In general, GA is required for seed development, which is determined through the analysis of GA-deficient mutants. In pea (Pisum sativum L.), the GAbiosynthesis mutant $l h$ has provided the best evidence for the physiological role of GA in seed development. The LH protein, which encodes ent-kaurene oxidase (KO), is required for the three-step oxidation of ent-kaurene to ent-kaurenoic acid as an early step in the GA biosynthesis pathway altering seed development [45]. KS (ent-kaurene synthase) and $\mathrm{KO}$ are key enzymes in the GA biosynthetic pathway, and both are encoded by a single gene in soybean. $\mathrm{GA}_{2} \mathrm{Ox}\left(\mathrm{GA}_{2}\right.$-oxidase) plays an important role in the last step of GA biosynthesis and catalyses the inactivation of GAs by conversion to bioactive GAs, thereby affecting the amount of bioactive GAs. Overexpression of $\mathrm{GA}_{2} \mathrm{Ox}$ genes results in reduced GA level in Arabidopsis [46], rice [47], and poplar [48]. DELLA-domain proteins are transcriptional regulators that repress GA responses, and these proteins are rapidly degraded in response to GA. In this study, one $K O$ gene and one $K S$ gene were downregulated in the cco mutant, suggesting that an early step in GA biosynthesis was blocked (Additional file 5). In addition, four $G A_{2} O x$ genes and three DELLA genes were up-regulated, suggesting that the rate of conversion of inactive GAs to bioactive GAs was limited for the $c c o$ mutant (Figure 7 and Additional file 5).

ABA not only plays an important role in the stress responses and plant tolerance, but also controls seed development and germination. The enzyme 9-cis-epoxycarotenoid dioxygenase (NCED) is involved in a rate-limiting step for ABA biosynthesis. Recently, Martínez-Andújar et al. reported that the NCED6 induction enhanced seed dormancy in Arabidopsis [49]. Here, we showed that NCED genes were up-regulated in the $c c o$ mutant, suggesting that ABA synthesis was promoted. However, another gene encoding abscisic acid $8^{\prime}$-hydroxylase, which catalyses a key step in ABA catabolism, was also up-regulated (Additional file 6). Snf1-related protein kinases (SnRK2s) are key positive regulators in ABA signal transduction pathway. In the absence of $A B A$, protein phosphatases type 2C (PP2Cs) physically interact with SnRK2s and negatively regulate ABA responses. However, interactions between PP2Cs and SnRK2s are disrupted when an ABA molecule binds to PYRABACTIN RESISTANCE1 (PYR1)/ PYR1-LIKE (PYL)/REGULATORY COMPONENTS OF ABA RECEPTORS (RCAR) receptors, leading to structural changes in these receptors. When SnRK2s are released from PP2C inhibition, their downstream targets, including ABA-Responsive Element Binding Factors (ABFs), are activated [50]. Fujii et al. constructed an Arabidopsis line carrying mutations in all 10 members of the SnRK2 family. The decuple mutant snrk2.1/2/3/4/5/6/ $7 / 8 / 9 / 10($ srk $2 g / d / e / a / h / e / f / c / j / b)$ was defective in gene 


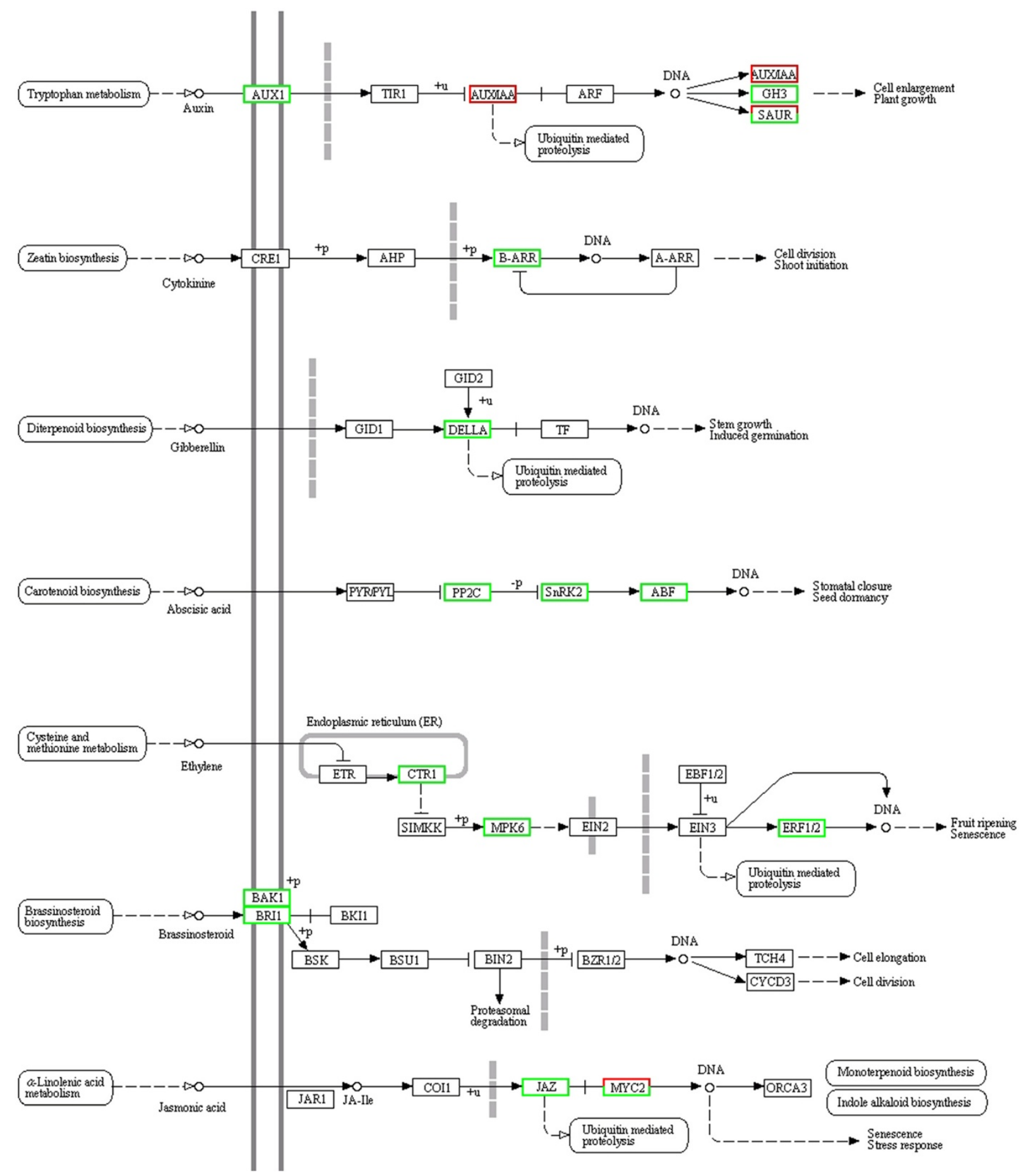

Figure 7 Transcriptional changes in plant hormone signal transduction pathways. Differentially expressed genes were mapped to the KEGG pathway database. Genes showing up-regulation and down-regulation are boxed in green and red, respectively. 
regulation and $A B A$ accumulation [51]. In our data, the $P P 2 C s, S n R K 2$ and $A B F$ genes were up-regulated in the cco mutant (Figure 7). In summary, ABA biosynthesis, catabolism and signalling were enhanced in cco.

In addition to auxin, GA and ABA, the pathways of other hormone biosynthesis and signal transduction (such as CK, ET, BR, and JA) were also influenced in the cco mutant. CK and ET, as major classes of plant hormone, are involved in various aspects of plant development, including organ development [52,53]. In cco, we found two genes encoding adenosine-phosphate isopentenyltransferase (IPT), a rate-limiting enzyme in CK biosynthesis, expressed at significantly higher levels [54,55] (Additional file 7). In CK signalling, type-B ARABIDOPSIS RESPONSE REGULATORS (ARRs) are transcriptional activators that regulate cytokinin targets, including type-A ARRs [56]. We observed the up-regulation of two type-B ARR genes (Figure 7). Aminocyclopropane-carboxylate oxidase (ACO), an "ethylene-forming enzyme" (EFE), catalyses the final step in ethylene biosynthesis, converting 1-amino-cyclopropane-1-carboxylic acid (ACC) into ethylene, $\mathrm{CO} 2$, and cyanide. Four $A C O$ genes were up-regulated in cco (Additional file 8). Antisense constructs of $A C O$ genes can notably reduce ET production [57]. ET signalling was also influenced in the cco mutant, and the expression levels of ethylene-related genes encoding serine/threonine-protein kinase (CTR1), mitogenactivated protein kinase 6 (MPK6), and ethylene-responsive transcription factor (ERF1/2) were altered in cco (Figure 7 and Additional file 8 ). BRs regulate embryonic and postembryonic development, adult homeostasis and expression of numerous genes [58]. Plant steroid hormones are recognised by brassinosteroid-insensitive 1 (BRI1), whose kinase activity is activated by exogenous BR application [59]. We also found that the expression levels of thirteen $B R 1$ and four BRI1 Associated receptor Kinase 1 (BAK1) genes in cco were higher than those in WT (Figure 7). JA regulates plant growth and stress responses, biosynthetic pathway of which starts with $\alpha$-linolenic acid [60]. The DEGs in JA biosynthesis and signalling pathway included those that encode phospholipase A1, 12-oxo-phytodienoic acid reductase, lipoxygenase, JASMONATE-ZIM (JAZ) and MYC2 proteins [61] ( Additional file 9 and Figure 7).

Auxin, BR, CK and GA are generally considered major developmental growth regulators, whereas ABA, ET and JA are involved in stress responses, all of these hormones converge on auxin [62,63]. Auxin is not only a hormone but also a morphogen. Auxin accumulation is controlled by auxin biosynthesis and metabolism, the conjugation/deconjugation of active auxin to/from its inactive conjugated form and auxin transport [64-66]. Auxin maxima and activity have been implicated in embryo development and patterning [37]. In $c c o$, the free IAA level and auxin polar transport were affected. Polar auxin transport promotes the formation of local auxin maxima and gradients within tissues that play important roles in auxin action, such as the patterns of cell division and differentiation in the root meristem [67] and the establishment of cotyledons [26,29]. Plants show directional growth in response to environmental stimuli such as light or gravity (tropisms). It was found that auxin played an important role in tropisms [68] and unequal auxin distribution across organs may lead their bending [69]. The cotyledons of cco showed outward bending, which allowed us to hypothesise that PAT might cause an auxin imbalance between the abaxial and adaxial side in cotyledons of the cco mutant, resulting in the adaxial side developing faster than the abaxial side. However, in soybean embryogenesis, the endosperm surrounds the embryo and cotyledons, the curled cotyledons observed in the cco mutant might reflect the fetter of the endosperm. Many signals can regulate auxin polar transport including stress, protein phosphorylation, reactive oxygen, flavonols and other hormones [70-73], which among these signals affect(s) PAT in cco require further investigation. In $c c o$, only the shape of cotyledons not including leaves was abnormal, indicating that signal(s) or mutated genes regulating PAT were specific for cotyledon initiation and growth.

GA and ABA have mainly been implicated in later stages of embryo development, acting antagonistically in the regulation of seed germination [74]. Compared with WT, cco showed higher IAA and ABA levels, whereas a lower $\mathrm{GA}_{3}$ level. Several reports indicate that auxin and GA overlap in the regulation of multiple aspects of plant development, and there is positive cross-talk interaction between them. Willige et al. have reported that a mutant deficient in GA biosynthesis and signalling showed a reduced PIN protein level, which could be recovered by exogenous GA application. Moreover, GA could interact with PIN1 and promote cotyledon differentiation [75]. Auxin can also induce GA biosynthesis. Root cells with lower auxin level have reduced GA biosynthesis [76,77]. Interestingly, our results demonstrated a lower $\mathrm{GA}_{3}$ but higher IAA level in $c c o$ seeds. But beyond that, $c c o$ seeds had higher ABA content, which might correlate with lower $\mathrm{GA}_{3}$ level and may be the reason for lower germination rate in $c c o$. Consistently, ABA negatively regulates bioactive GA level during seed germination.

\section{Conclusions}

Multiple phytohormone biosynthesis and signalling pathways are reprogrammed in the cco mutant, which is consistent with our HPLC analysis. Our results indicate that curled-cotyledons and reduced seed germination rate may result from the biosynthesis and signalling disorder of multiple phytohormones. Accumulating evidence supports the idea that hormones not only act in a linear pathway but also function in a complex network of feedback 
regulation and cross talk within or between different hormone pathways. At present, the studies of molecular mechanisms underlying hormonal cross talk mainly focus on postembryonic development, including the roots, shoots and leaves. However, adequate models of the hormonal network involved in cotyledon development are not well established in plants. Analysis of $c c o$ may provide a promising frame for further studies of hormone interactions and offer the potential discovery of new genes controlling cotyledon development in plants.

\section{Methods}

\section{Plant materials}

Soybean seeds from wild type cultivars Nannong 94-16 and its cotyledon mutant $c c 0$ were provided by Soybean Research Institute, Nanjing Agricultural University, China. The seeds were cultivated in field under natural conditions in Nanjing, China. Total RNA was extracted from the WT and cco tissues, including the roots, stems, leaves, flowers and seeds, which were frozen in liquid nitrogen and stored at $-80^{\circ} \mathrm{C}$ until further analysis.

\section{Plant paraffin section}

For paraffin sectioning, wild type and mutant seeds at various developmental stages were fixed in Carnoy's fluid (ethanol/glacial acetic acid, 3:1) at room temperature followed by dehydration in a graded ethanol series, staining with $1 \%$ haematoxylin solution, and clearance with xylene. The seeds were embedded in paraffin and sectioned at 10 to $15 \mu \mathrm{m}$ (Leica, RM2135).

\section{Semi-quantitative RT-PCR and quantitative RT-PCR (qRT-PCR)}

To analyse the gene expression in soybean, total RNA was extracted with the Plant RNA Extract Kit (TianGen, Beijing, China) according to the manufacturer's instructions. PCR amplification was performed with PrimeScript $1^{\text {st }}$ Strand cDNA Synthesis Kit (Takara). qRT-PCR was performed as described previously [78]. The Gmtubulin (GenBank no.AY907703) and Gmactin (GenBank no. XM_003531354) genes were used as reference genes for qRT-PCR and semi-quantitative RT-PCR, respectively. The primers used in this study were provided in Additional file 10.

\section{Extraction of endogenous free IAA, GA $A_{3}, A B A$ and HPLC analysis}

A total of $0.5 \mathrm{~g}$ fresh seeds at 7DAF from at least 10 individual lines of WT and $c c o$ were ground in liquid nitrogen, phytohormones were extracted in 10 milliliter cold $80 \%(\mathrm{v} / \mathrm{v})$ methanol and $1 \%(\mathrm{w} / \mathrm{v})$ polyvinylpyrrolidone was added as an antioxidant. After $24 \mathrm{~h}$ of incubation at $4^{\circ} \mathrm{C}$, the samples were centrifuged for $15 \mathrm{~min}$ at $12,000 \mathrm{~g}$ at $4^{\circ} \mathrm{C}$. The supernatant was retained, and the pellet was re-extracted with $10 \mathrm{ml}$ of cold $80 \%(\mathrm{v} / \mathrm{v})$ methanol and recentrifuged as indicated above. The supernatants were pooled. After evaporation under a $\mathrm{N}_{2}$ gas flow, the $\mathrm{pH}$ of the remaining water phase was adjusted to 3.0. IAA, $\mathrm{GA}_{3}$ and $\mathrm{ABA}$ purification was performed using acetyl acetate. The acetyl acetate was also evaporated under an $\mathrm{N}_{2}$ stream. The resulting dried precipitate was collected in $0.5 \mathrm{ml}$ of $100 \%$ methanol, filtered through $0.45 \mu \mathrm{m}$ filter membrane and submitted to HPLC (Shimadzu, Kyoto, Japan) analysis. HPLC analysis was performed using an Alltima C18 column $(4.6 \mathrm{~mm} \times 250 \mathrm{~mm})$. The IAA and $\mathrm{ABA}$ concentration was determined at $254 \mathrm{~nm}$, and the $\mathrm{GA}_{3}$ concentration was determined at $210 \mathrm{~nm}$. Hormone measurements were conducted in triplicate and subjected to statistical analysis.

\section{IAA sensibility assay}

WT and cco seeds were germinated in moistened vermiculite at $30^{\circ} \mathrm{C}$. After $72 \mathrm{~h}$, the seedlings were sprayed with $2.5 \times 10^{-3} \mathrm{M}$ 2,4-D solution ( $\mathrm{pH}$ 6). The intact hypocotyls were then segregated from the seedlings and incubated in $10 \mathrm{ml}$ of incubation medium in the absence or presence of $5 \times 10^{-5} \mathrm{M}$ and $2 \times 10^{-4} \mathrm{M} 2,4-\mathrm{D}(\mathrm{pH} 6$ ) at $30^{\circ} \mathrm{C}$ with continuous shaking. At 0.5 and $1 \mathrm{~h}$, the 1.2$\mathrm{cm}$ sections obtained from the elongating region of the hypocotyls $(0.5-1.7 \mathrm{~cm}$ below the cotyledons) were excised. The hypocotyl sections were rinsed in deionised distilled $\mathrm{H}_{2} \mathrm{O}$ and immediately frozen in liquid $\mathrm{N}_{2}$. The sections were stored at $-70^{\circ} \mathrm{C}$ until further use for RNA extraction.

\section{cDNA library construction for RNA-Seq and sequencing}

Beads with oligo (dT) were used to isolate poly (A) mRNA after total RNA was collected. Fragmentation buffer was added to digest the mRNA to generate short fragments. These short fragments were used as templates, and random hexamer-primers were used to synthesise first-strand cDNA. Second-strand cDNA was synthesised in a reaction containing buffer, dNTPs, RNase $\mathrm{H}$ and DNA polymerase I. Short fragments were purified using the QIAquick PCR extraction kit (QIAGEN) and eluted in EB buffer for end preparation and poly (A) addition. Subsequently, the short fragments were ligated with sequencing adaptors. For PCR amplification, we selected suitable fragments to use as templates based on the results of agarose gel electrophoresis. The libraries were sequenced using the Illumina HiSeq $^{\text {Tw }} 2000$ system.

\section{Raw read filtering and mapping to the reference genome and genes sequences}

Dirty raw reads were discarded via the following four steps: 1 . reads with adaptors were removed; 2 . reads with unknown nucleotides larger than 5\% were removed; 3 . reads with low quality (more than half of the base quality 
less than 5) were removed; 4 . clean reads were obtained. Clean reads were mapped to the reference genome and genes sequences respectively using SOAPaligner/soap2. Mismatches with no more than $\mathrm{m}$ ( $\mathrm{m}$ default is 5 ) bases were used in the alignment. All subsequent analyses were based on clean reads.

\section{Gene expression annotation}

Gene coverage is the percentage of a gene covered by reads. This value equals the ratio of the number of bases in a gene covered by unique mapping reads to the number of total bases in that gene. The UniGene expression was calculated using the RPKM method (Reads Per kb per Million reads). The RPKM method eliminated the influence of different gene lengths and sequencing discrepancies on the gene expression calculations. Therefore, the calculated gene expression could be used to directly compare differences in gene expression between the samples. If there was more than one transcript for a gene, the longest transcript was used to calculate the expression level and coverage.

\section{Differentially expressed gene analysis}

Using "The significance of digital gene expression profiles" [79], we identified differentially expressed genes between WT and the cco mutant based on the following criteria: $(\mathrm{FDR})<0.001$ and $\mid \log _{2}$ Ratio $\mid \geq 1$.

\section{GO enrichment and KEGG pathway analysis}

The differentially expressed genes were subjected to GO enrichment analysis. The analysis first mapped all DEGs to GO terms in the database (http://www.geneontology.org/), calculating gene numbers for every term, followed by the ultra-geometric test to identify significantly enriched GO terms in DEGs compared with the genome background. KEGG Pathway (http://www.genome.jp/kegg/pathway.html) enrichment analysis was used to identify significantly enriched metabolic or signal transduction pathways in DEGs compared with the whole genome background.

\section{Availability of supporting data}

The RNA-seq data of this article have been deposited in the Gene Expression Omnibus (GEO) Database, with the following access number: GSE58354 (http://www.ncbi.nlm. nih.gov/geo/query/acc.cgi?acc=GSE58354). Other supporting data are included as additional files.

\section{Additional files}

Additional file 1: 1093 differentially expressed genes between WT and $c$ co.

Additional file 2: Semi-quantitative RT-PCR analysis of 15 selected genes. (A) and (B): semi-quantitative RT-PCR analysis of 15 selected genes in WT and cco pods at 7 DAF. (C): semi-quantitative RT-PCR analysis of 4 of the 15 selected genes in WT and cco seeds at 7 and 15 DAF. 1: WT seeds at 7 DAF; 2: cco seeds at 7 DAF; 3: WT seeds at 15 DAF; 4: cco seeds at 15 DAF

Additional file 3: Differentially expressed genes in hormone biosynthesis and signal transduction pathways.

Additional file 4: Transcriptional changes in tryptophan metabolism. Differentially expressed genes from RNA-Seq were mapped to the KEGG pathway database. The up-regulated genes in cco are boxed in green.

Additional file 5: Transcriptional changes in zeatin biosynthesis. The up-regulated and down-regulated genes in cco are boxed in green and red, respectively.

Additional file 6: Transcriptional changes in diterpenoid biosynthesis. The up-regulated and down-regulated genes in cco are boxed in green and red, respectively.

Additional file 7: Transcriptional changes in carotenoid biosynthesis pathway. The up-regulated and down-regulated genes in cco are boxed in green and red, respectively.

Additional file 8: Transcriptional changes in cysteine and methionine metabolism pathway. The up-regulated and down-regulated genes in cco are boxed in green and red, respectively.

Additional file 9: Transcriptional changes in a-linolenic acid metabolism pathway. The up-regulated and down-regulated genes in cco are boxed in green and red, respectively.

Additional file 10: The list of primers used in this article.

\section{Competing interests}

The authors declare that they have no competing interests.

\section{Authors' contributions}

DYY, FH and QSC conceived the study. GXS performed the statistical analyses for RNA-seq. GXS, YG, GLX and JJY performed all of the experimental work in this paper. GXS, FH, ZBH and DYY wrote the manuscript. All authors read and approved the final manuscript.

\section{Acknowledgments}

This study was supported in part by funding from the National Basic Research Program of China (973 Program) (2010CB125906), the Key Transgenic Breeding Program of China (2013ZX08004-003), and the National Natural Science Foundation of China $(31171573,31371644)$.

Received: 18 January 2014 Accepted: 18 June 2014

Published: 21 June 2014

\section{References}

1. Bils RF, Howell RW: Biochemical and cytological changes in developing soybean cotyledons. Crop Sci 1963, 3:304-308.

2. Goldberg RB, Paiva G, Adegari RY: Plant embryogenesis: zygote to seed. Science 1994, 266:605-614.

3. Abe M, Katsumata H, Komeda Y, Takahashi T: Regulation of shoot epidermal cell differentiation by a pair of homeodomain proteins in Arabidopsis. Development 2003, 130:635-643.

4. Aida M, Ishida T, Tasaka M: Shoot apical meristem and cotyledon formation during Arabidopsis embryogenesis: interaction among the CUP-SHAPED COTYLEDON and SHOOT MERISTEMLESS genes. Development 1999, 126:1563-1570.

5. Izhakia A, Bowman JL: KANADI and class III HD-Zip gene families regulate embryo patterning and modulate auxin flow during embryogenesis in Arabidopsis. Plant Cell 2007, 19:495-508.

6. Long JA, Woody S, Poethig S, Meyerowitz EM, Barton MK: Transformation of shoots into roots in Arabidopsis embryos mutant at the TOPLESS locus. Development 2002, 129:2297-2306.

7. Long JA, Ohno C, Smith ZR, Meyerowitz EM: TOPLESS regulates apical embryonic fate in Arabidopsis. Science 2006, 312:1520-1523.

8. Weir I, Lu J, Cook H, Causier B, Schwarz-Sommer Z, Davies B: CUPULIFORMIS establishes lateral organ boundaries in Antirrhinum. Development 2003, 131:915-922. 
9. Keddie JS, Carroll BJ, Thomas CM, Reyes MEC, Klimyuk V, Holtan H, Gruissem W, Jones JDG: Transposon tagging of the defective embryo and meristems gene of tomato. Plant Cell 1998, 10:877-887.

10. Avasarala S, Yang J, Caruso JL: Production of phenocopies of the lanceolate mutant in tomato using polar auxin transport inhibitors. J Exp Biol 1996, 47:709-712.

11. Al-Hammadi ASA, Sreelakshmi Y, Negi S, Siddiqi I, Sharma R: The polycotyledon mutant of tomato shows enhanced polar auxin transport. Plant Physiol 2003, 133:113-125.

12. Liu CM, Johnson S, Gregorio SD, Wang TL: Single cotyledon(sic) mutants of pea and their significance in understanding plant embryo development. Dev Genet 1999, 25:11-22.

13. Chandler JW: Cotyledon organogenesis. J Exp Bio/ 2008, 59:2917-2931

14. Larsson E, Sundström JF, Sitbon F, Arnold SV: Expression of PaNACO1, a picea abies CUP-SHAPED COTYLEDON orthologue, is regulated by polar auxin transport and associated with differentiation of the shoot apical meristem and formation of separated cotyledons. Ann Bot 2012, 110:923-934.

15. Aida M, Ishida T, Fukaki H, Fujisawa H, Tasaka M: Genes involved in organ separation in Arabidopsis: an analysis of the cup-shaped cotyledon mutant. Plant Cell 1997, 9:841-857.

16. Roemen CWV, Mordhorst AP, Albrecht C, Kwaaitaal MACJ, De Vries SC: The CUP-SHAPED COTYLEDON3 gene is required for boundary and shoot meristem formation in Arabidopsis. Plant Cell 2003, 15:1563-1577.

17. Spinelli SV, Martin AP, Viola IL, Gonzalez DH, Palatnik JF: A mechanistic link between STM and CUC1 during Arabidopsis development. Plant Physiol 2011, 156:1894-1904.

18. Byrne ME, Barley R, Curtis M, Arroyo JM, Dunham M, Hudson A, Martienssen RA: Asymmetric leaves 1 mediates leaf patterning and stem cell function in Arabidopsis. Nature 2000, 408:967-971.

19. Belles-Boix PE, Hamant O, Witiak SM, Morin H, Véronique JT: KNAT6: an Arabidopsis homeobox gene involved in meristem activity and organ separation. Plant Cell 2006, 18:1900-1907.

20. Oliva $M$, Farcot $E$, Vernoux T: Plant hormone signaling during development: insights from computational models. Curr Opin Plant Biol 2013, 16:19-24.

21. Nishimura C, Ohashi Y, Sato S, Kato T, Tabata S, Ueguchi C: Histidine kinase homologs that act as cytokinin receptors possess overlapping functions in the regulation of shoot and root growth in Arabidopsis. Plant Cell 2004, 16:1365-1377.

22. Cheng $Y$, Dai $X$, Zhao $Y$ : Auxin biosynthesis by the YUCCA flavin monooxygenases controls the formation of floral organs and vascular tissues in Arabidopsis. Gene Dev 2006, 20:1790-1799.

23. Cheng $Y$, Dai $X$, Zhao $Y$ : Auxin synthesized by the YUCCA flavin monooxygenases is essential for embryogenesis and leaf formation in Arabidopsis. Plant Cell 2007, 19:2430-2439.

24. Liu CM, Xu ZH, Chua NH: Auxin polar transport is essential for the establishment of bilateral symmetry during early plant embryogenesis. Plant Cell 1993, 5:621-630.

25. Ramesar-Fortner NS, Yeung EC: Tri-iodobenzoic acid affects shoot apical meristem formation and function in zygotic embryos of Brassica napus cv. Topas. Ca J Bot 2001, 79:265-273.

26. Furutani M, Vernoux T, Traas J, Kato T, Tasaka M, Aida M: PIN-FORMED1 and PINOID regulate boundary formation and cotyledon development in Arabidopsis embryogenesis. Development 2004, 131:5021-5030.

27. Larsson E, Sitbon F, Ljung K, Arnold SV: Inhibited polar auxin transport results in aberrant embryo development in Norway spruce. New Phytol $2008,177: 356-366$

28. Friml J, Vieten A, Sauer M, Weijers D, Schwarz H, Hamann T, Offringa R, Jurgens G: Efflux-dependent auxin gradients establish the apical-basal axis of Arabidopsis. Nature 2003, 426:147-153.

29. Treml BS, Winderl S, Radykewicz R, Herz M, Schweizer G, Hutzler $P$ Glawischnig E, Ruiz RAT: The gene ENHANCER OF PINOID controls cotyledon development in the Arabidopsis embryo. Development 2005 132:4063-4074.

30. Aderkas PV: In vitro phenotypic variation in larch cotyledon number. Int $J$ Plant Sci 2002, 163:301-307.

31. Chaudhury AM, Letham S, Craig S, Dennis E: Amp1: a mutant with high cytokinin levels and altered embryonic pattern, faster vegetative growth, constitutive photomorphogenesis and precocious flowering. Plant J 1993, 4:907-916.
32. Banno H, Ikeda Y, Niu QW, Chua NH: Overexpression of Arabidopsis ESR1 induces initiation of shoot regeneration. Plant Cell 2001, 13:2609-2618.

33. Ikeda Y, Banno H, Niu QW, Howell SH, Chua NH: The ENHANCER OF SHOOT REGENERATION 2 gene in Arabidopsis regulates CUP-SHAPED COTYLEDON 1 at the transcriptional level and controls cotyledon development. Plant Cell Physiol 2006, 47:1443-1456.

34. Yu JJ, Han SY, Shi GX, Yu DY: Characterization of a novel curled-cotyledons mutant in soybean [Glycine max (L.) Merr.]. Afr J Biotechnol 2012, 11:14889-14898.

35. West ALM, Harada JJ: Embryogenesis in higher plants. Plant Cell 1993, 5:1361-1369.

36. Vanneste1 S, Friml J: Auxin: a trigger for change in plant development. Cell 2009, 136:1005-1016.

37. Möller B, Weijers D: Auxin control of embryo patterning. Cold Spring Harb Perspect in Biol 2009, 1:a001545.

38. Joint Genome Institute/Phytozome/. http://www.phytozome.net/soybean.php.

39. Asakura T, Tamura T, Terauchi K, Naikawa T, Yagasaki K, Ishimaru Y, Abe K: Global gene expression profiles in developing soybean seeds. Plant Physiol Bioch 2012, 52:147-153.

40. Stepanova AN, Hoyt JM, Hamilton AA, Alonso JM: A link between ethylene and auxin uncovered by the characterization of two root-specific ethylene-insensitive mutants in Arabidopsis. Plant Cell 2005, 17:2230-2242.

41. Morino K, Matsuda F, Miyazawa H, Sukegawa A, Miyagawa H, Wakasa K: Metabolic profiling of tryptophan-overproducing rice calli that express a feedback-insensitive a subunit of anthranilate synthase. Plant Cell Physiol 2005, 46:514-521.

42. Mano $Y$, Nemoto $K$ : The pathway of auxin biosynthesis in plants. J Exp Biol 2012, 63:2853-2872.

43. Abel S, Theologis A: Early genes and auxin action. Plant Physiol 1996, 111:9-17.

44. Ulmasov T, Murfett J, Hagen G, Guilfoyle TJ: Aux/IAA proteins repress expression of reporter genes containing natural and highly active synthetic auxin response elements. Plant Cell 1997, 9:1963-1971.

45. Swain SM, Reid JB, Kamiya Y: Gibberellins are required for embryo growth and seed development in pea. Plant J 1997, 12:1329-1338.

46. Schomburg FM, Bizzell CM, Lee DJ, Zeevaart JAD, Amasinoa RM: Overexpression of a novel class of gibberellin 2-oxidases decreases gibberellin levels and creates dwarf plants. Plant Cell 2003, 15:151-163.

47. Sakamoto T, Morinaka Y, Ishiyama K, Kobayashi M, Itoh H, Kayano T, Iwahori S, Matsuoka M, Tanaka H: Genetic manipulation of gibberellin metabolism in transgenic rice. Nat Biotechnol 2003, 21:909-913.

48. Busov VB, Meilan R, Pearce DW, Ma C, Rood SB, Strauss SH: Activation tagging of a dominant gibberellin catabolism gene (GA2-oxidase) from poplar that regulates tree stature. Plant Physiol 2003, 132:1283-1291.

49. Martínez-Andújar C, Ordiz MI, Huang ZL, Nonogaki M, Beachy RN, Nonogaki $\mathrm{H}$ : Induction of 9-cis-epoxycarotenoid dioxygenase in Arabidopsis thaliana seeds enhances seed dormancy. Proc Natl Acad Sci U S A 2011, 108:17225-17229.

50. Klingler JP, Batelli G, Zhu JK: ABA receptors: the START of a new paradigm in phytohormone signalling. J Exp Biol 2010, 61:3199-3210.

51. Fujii $\mathrm{H}$, Verslues PE, Zhu JK: Arabidopsis decuple mutant reveals the importance of SnRK2 kinases in osmotic stress responses in vivo. Proc Natl Acad Sci U S A 2011, 108:1717-1722.

52. Merchante C, Alonso JM, Stepanova AN: Ethylene signaling: simple ligand, complex regulation. Curr Opin Plant Biol 2013, 16:554-560.

53. Werner T, Schmülling T: Cytokinin action in plant development. Curr Opin Plant Biol 2009, 12:527-538.

54. Ma QH, Wang XM, Wang ZM: Expression of isopentenyl transferase gene controlled by seed-specific lectin promoter in transgenic tobacco influences seed development. J Plant Growth Regul 2008, 27:68-76.

55. Brugière N, Humbert $\mathrm{S}$, Rizzo N, Bohn J, Habben JE: A member of the maize isopentenyl transferase gene family, Zea mays isopentenyl transferase 2 ( $Z m I P T 2)$, encodes a cytokinin biosynthetic enzyme expressed during kernel development. Plant Mol Biol 2008, 67:215-229.

56. To JPC, Kieber JJ: Cytokinin signaling: two-components and more. Trends Plant Sci 2008, 13:85-92.

57. Hamilton AJ, Lycett GW, Grierson D: Antisense gene that inhibits synthesis of the hormone ethylene in transgenic plants. Nature 1990, 346:284-287.

58. Chandler JW, Cole M, Flier A, Werr W: BIM1, a bHLH protein involved in brassinosteroid signalling, controls Arabidopsis embryonic patterning via 
interaction with DORNRÖSCHEN and DORNRÖSCHEN-LIKE. Plant Mol Biol 2009, 69:57-68.

59. Li JM, Jin H: Regulation of brassinosteroid signaling. Trends Plant Sci 2006, 12:37-41.

60. León J, Sánchez-Serrano JJ: Molecular biology of jasmonic acid biosynthesis in plants. Plant Physiol Bioch 1999, 37:373-380.

61. Katsir L, Chung HS, Koo AJK, Howe GA: Jasmonate signaling: a conserved mechanism of hormone sensing. Curr Opin Plant Biol 2008, 11:428-435.

62. Hardtke CS, Depuyd S: Hormone signalling crosstalk in plant growth regulation. Curr Biol 2011, 21:365-373.

63. Jürgens $\mathrm{G}$, Wolters $\mathrm{GH}$ : Survival of the flexible: hormonal growth control and adaptation in plant development. Nature 2009, 10:305-317.

64. Chandler JM: Local auxin production: a small contribution to a big field. Bioessays 2009, 31:60-70

65. Ikeda Y, Men S, Fischer U, Stepanova A, Alonso J, Ljung K: Local auxin biosynthesis modulates gradient-directed planar polarity in Arabidopsis. Nat Cell Biol 2009, 11:731-738.

66. Petrásek J, Friml J: Auxin transport routes in plant development. Development 2009, 136:2675-2688.

67. Friml J: Auxin transport-shaping the plant. Curr Opin Plant Biol 2003, 6:7-12.

68. Went FW: Reflections and speculations. Annu Rev Plant Physiol 1974, 25:1-26.

69. Tanaka H, Dhonukshe P, Brewer PB, Friml J: Spatiotemporal asymmetric auxin distribution: a means to coordinate plant development. Cell Mol Life Sci 2006, 63:2738-2754.

70. Chen RJ, Baluška F: Regulation of polar auxin transport by protein phosphorylation. In Polar Auxin Transpot. Volume 4. Edited by Martínez, et al. Spain: Universitat Autònoma de Barcelona; 2013:81-101.

71. Chen RJ, Baluška F: Control of auxin transport by reactive oxygen and nitrogen species. In Polar Auxin Transpot. Volume 5. Edited by FernándezMarcos, et al. Spain: Universidad de Salamanca; 2013:103-117.

72. Kuhn BM, Gersler M, Bigler L, Ringli C: Flavonols accumulate asymmetrically and affect auxin transport in Arabidopsis. Plant Physiol 2011, 156:585-595.

73. Chen RJ, Baluška F: Hormonal control of polar auxin transport. In Polar Auxin Transpot. Volume 6. Edited by Sun, et al. China: Chinese Academy of Sciences; 2013:119-133.

74. Holdsworth MJ, Bentsink L, Soppe WJ: Molecular networks regulating Arabidopsis seed maturation, after-ripening, dormancy and germination. New Phytol 2008, 179:33-54.

75. Willige BC, Isono E, Richter R, Zourelidou M, Schwechheimer C: Gibberellin regulates PIN-FORMED abundance and is required for auxin transportdependent growth and development in Arabidopsis thaliana. Plant Cell 2011, 23:2184-2195.

76. Frigerio M, Alabadí D, Pérez-Gómez J, García-cárcel L, Phillips AL, Hedden P, Blázquez MA: Transcriptional regulation of gibberellin metabolism genes by auxin signaling in Arabidopsis. Plant Physiol 2006, 142:553-563.

77. Dorcey E, Urbez C, Blazquez MA, Carbonell J, Perez-Amador MA: Fertilization-dependent auxin response in ovules triggers fruit development through the modulation of gibberellin metabolism in Arabidopsis. Plant J 2009, 58:318-332.

78. Yin ZT, Meng FF, Song HN, Wang XL, Xu XM, Yu DY: Expression quantitative trait loci analysis of two genes encoding rubisco activase in soybean. Plant Physiol 2010, 152:1625-1637.

79. Audic S, Claverie JM: The significance of digital gene expression profiles. Genome Res 1997, 7:986-995.

doi:10.1186/1471-2164-15-510

Cite this article as: Shi et al:: RNA-Seq analysis reveals that multiple phytohormone biosynthesis and signal transduction pathways are reprogrammed in curled-cotyledons mutant of soybean [Glycine max (L.) Merr.]. BMC Genomics 2014 15:510.

\section{Submit your next manuscript to BioMed Central and take full advantage of:}

- Convenient online submission

- Thorough peer review

- No space constraints or color figure charges

- Immediate publication on acceptance

- Inclusion in PubMed, CAS, Scopus and Google Scholar

- Research which is freely available for redistribution

Submit your manuscript at www.biomedcentral.com/submit
C Biomed Central 